\title{
Avaliação química e sensorial da farinha de resíduo de tilápias na forma de sopa
}

\author{
Chemical and sensorial evaluation of fish meal in soup
}

\author{
Flávia Braidotti STEVANATO ${ }^{1}$, Maria Eugênia PETENUCCI ${ }^{1}$, Makoto MATSUSHITA ${ }^{1}$, \\ Michele Cristiane MESOMO ${ }^{1}$, Nilson Evelázio de SOUZA ${ }^{1}$, Jeane Eliete Laguila VISENTAINER ${ }^{1}$, \\ Vanessa Vivian de ALMEIDA ${ }^{1}$, Jesui Vergilio VISENTAINER ${ }^{1 *}$
}

\section{Resumo}

As cabeças de tilápias são resíduos do processamento de peixes comumente descartados e não aproveitadas como alimento. Desta forma realizou-se um estudo sobre a composição química e de ácidos graxos na farinha obtida a partir de cabeças de tilápias. Ainda foram realizadas avaliações sensorial (caldo e sopa) e química e de ácidos graxos na sopa elaborada com a farinha. Os objetivos foram avaliar a composição e aceitação dos produtos elaborados com farinha, visando o aproveitamento na alimentação humana, especialmente para a merenda escolar. Os resultados obtidos foram de elevados teores de proteína $(38,4 \%)$, cinzas $(19,4 \%)$ e lipídios $(35,5 \%)$ na farinha. A sopa apresentou elevada aceitação pelas crianças do ensino fundamental e a composição de ácidos graxos do conteúdo lipídico indicou a presença de diversos ácidos ômega-3, especialmente os ácidos alfa-linolênico (LNA), eicosapentaenóico (EPA), docosahexaenócio (DHA) e excelente razão AGPI/AGS. Todos estes parâmetros evidenciam que a inclusão de cabeça de tilápia na forma de farinha é aceitável como alimento e constitui uma fonte nutritiva e benéfica para a saúde humana.

Palavras-chave: tilápia; cabeça; farinha; composição química; ácidos graxos.

\begin{abstract}
Fish heads are normally discarded during fish processing and are not used as food. This study therefore investigated the chemical and fatty acids composition of fish meal produced from tilapia heads. An evaluation was made of the sensorial, chemical and fatty acids characteristics of broth and soup containing this fish meal. The purpose of the study was to analyze the composition and acceptance of products containing fish meal, aiming at its use in human food, especially in school meals. The results indicated that the fish meal contained contents of protein (38.4\%), ashes (19.4\%) and lipids (35.5\%). The soup containing fish meal was well accepted by elementary schoolchildren. Moreover, the fatty acids composition of the lipid content indicated the presence of several omega-3 acids, especially alpha-linolenic acid (ALA), eicosapentaenoic acid (EPA), docosahexaenoic acid (DHA) and an excellent PUFA/SFA ratio. All these parameters indicate that fish meal made from discarded tilapia heads is an acceptable, nutritious and healthy source of food for humans.

Keywords: tilapia; head; fish meal; chemical composition; fatty acids.
\end{abstract}

\section{Introdução}

As tilápias (Oreochromis niloticus) são as espécies de peixes mais cultivadas e consumidas no Brasil nos últimos anos ${ }^{10}$. A preferência nacional de consumo da carne de tilápia pelos brasileiros é na forma de filé, e no processo (filetagem) de sua obtenção são gerados resíduos, comumente não aproveitados, e que são descartados, poluindo o meio ambiente.

Os resíduos de peixes incluem todas as sobras de processamento tais como: filetagem, salga, enlatamento, defumação, etc. Estes resíduos apresentam valores relativamente baixos e incluem cabeça, fígado, espinhas, pele, vísceras, etc. ${ }^{13,18,20}$.

Estima-se que, da captura mundial de pescado, aproximadamente $72 \%$ é utilizado no mercado de peixe fresco, congelado, empanado, enlatado, etc. Os $28 \%$ restantes ou são utilizados no preparo de rações ou são desperdiçados como resíduos ${ }^{7}$. No Brasil, aproximadamente $50 \%$ da biomassa capturada é descartada durante o processamento ${ }^{14}$.

Os resíduos (fígado, cabeça, carcaça) de pescado apresentam alto teor de proteínas, minerais e lipídios. O conteúdo lipídico apresenta conteúdos apreciáveis de ácidos graxos

Recebido para publicação em 28/8/2006

Aceito para publicação em 19/7/2007 (001828)

Departamento de Química, Universidade Estadual de Maringá,

Av. Colombo, 5790, CEP 87020-900, Maringá - PR, Brasil,

E-mail: jvvisentainer@uem.br

*A quem a correspondência deve ser enviada poliinsaturados, principalmente da série ômega-3 (n-3) e ômega-6 (n-6). Nestas séries de ácidos graxos, destacam-se os ácidos alfa-linolênico (LNA, 18:3n-3) e linoléico (LA, 18:2n-6). Estes ácidos são considerados estritamente essenciais, ou seja, não são sintetizados pelo organismo humano, sendo necessária a ingestão na dieta ${ }^{1}$.

O LA é precursor do ácido araquidônico (AA, 20:4n-6), componente utilizado na síntese de prostaglandinas e importante para o crescimento fetal ${ }^{1}$, e o LNA é precursor dos ácidos graxos de cadeia longa (AGCL) da série $\mathrm{n}-3$, tais como o ácido eicosapentaenóico (EPA, 20:5n-3) e docosahexaenóico (DHA, 22:6n-3). Estes ácidos se destacam por apresentarem diversos efeitos benéfícos à saúde humana, como diminuição dos riscos de doenças cardiovasculares ${ }^{6}$, diminuição nas taxas de colesterol no sangue ${ }^{17}$, prevenção de câncer $^{16}$ e benefícios à gravidez e saúde materno-infantil ${ }^{5}$.

Visando o aproveitamento de resíduos de pescado e conseqüentemente fornecer subsídios para um destino mais nobre (alimentação humana) para esta parte do peixe, realizaram-se estudos sobre o processamento, composição química e de ácidos graxos da cabeça de tilápia. Os estudos de composição foram realizados na farinha pura, em um caldo e em uma sopa formulados com a farinha. As aceitações destes produtos foram avaliadas por universitários e por crianças do ensino fundamental de escola pública. 


\section{Material e métodos}

\subsection{Preparo da farinha}

Um total de 36 cabeças de tilápia foram previamente lavadas e submetidas ao cozimento a vapor, por 25 minutos. As cabeças cozidas foram trituradas em moinho rosca sem fim de aço inox e pesadas, totalizando $5,70 \mathrm{~kg}$.

Em seguida foram dispostas em assadeiras e levadas ao forno de um fogão convencional, permanecendo por 4 horas a $180^{\circ} \mathrm{C}$, depois de resfriada a farinha foi peneirada em peneiras de aço inox de 14 mesh, obtendo-se assim a farinha de cabeça de tilápia seca. A farinha foi embalada em sacos de polietileno, o ar removido e posteriormente enrolada em papel alumínio. A farinha foi armazenada em geladeira $\left(4^{\circ} \mathrm{C}\right)$, para posteriores análises e utilização no preparo do caldo e da sopa.

\subsection{Preparo do caldo e sopa}

A partir da farinha foram preparados dois produtos: caldo, contendo a farinha, água e sal, e a sopa, contendo vários ingredientes. Foi ainda preparado um controle constituído de todos os ingredientes da sopa, exceto a farinha de cabeça de tilápia.

O caldo foi preparado para um volume final de 1,5 litros. Os ingredientes utilizados foram: 5 colheres (sopa) óleo de soja; 15 colheres (sopa) de farinha de cabeça de tilápia; 2 colheres (sopa) sal e 2 litros de água. O preparo seguiu-se da seguinte forma: em uma panela de alumínio, contendo óleo de soja, acrescentou-se a farinha de cabeça de tilápia, em seguida, colocou-se sal e água; e o tempo de cozimento foi de 30 minutos.

Os ingredientes usados para um volume final de 1,5 litros de sopa foram: 5 colheres (sopa) de óleo de soja; 15 colheres (sopa) de farinha de cabeça de tilápia; 2 colheres (sopa) sal; 2 litros de água; 20 colheres (sopa) arroz cozido; 15 colheres (sopa) polpa de tomate; 5 dentes de alho triturados; 7 colheres (sopa) cebola triturada; 5 tomates picados; 2 colheres (sopa) coentro; 1 colher (sopa) de cebolinha; 1 colher (sopa) de salsinha e 1 colher (sopa) de orégano.

O preparo da sopa seguiu-se da seguinte forma: em uma panela de alumínio, foram refogados o alho e a cebola em óleo de soja. Acrescentaram-se a farinha de cabeça de tilápia e o tomate (picado e sem pele). Após o parcial amolecimento do tomate, acrescentou-se água. Em seguida, foram colocados arroz, polpa de tomate e mais um pouco de água. Por fim, adicionaram-se, orégano, sal, pimenta do reino, coentro, cebolinha e salsinha. O tempo de cozimento foi de 30 minutos.

O preparo do controle foi elaborado nas mesmas condições da sopa, entretanto não foi adicionada a farinha de cabeça de tilápia e o volume final do controle foi de aproximadamente 1,5 litro.

\subsection{Análise sensorial}

Os produtos caldo e sopa foram avaliados sensorialmente por um painel de 100 provadores. A análise sensorial do caldo foi realizada com 50 universitários e a sopa com 50 alunos do ensino fundamental (faixa etária de 10 a 12 anos).

A análise sensorial foi baseada no método de estímulo simples com escala Hedônica de 9 pontos com os extremos 1 (desgostei muitíssimo) e 9 (gostei muitíssimo). Os resultados da aceitabilidade do caldo e da sopa foram transformados de acordo com a fórmula: $\mathrm{Y}=(\mathrm{x}+0,5)^{1 / 2}$, cujos valores de $\mathrm{x}$ variam de 1 a 9 e desta forma os resultados de $Y$ estabelecem um intervalo de 3,08 (para a nota máxima $\mathrm{x}=9$ ) a 1,22 (para a nota mínima de $\mathrm{x}=1)^{3}$.

\subsection{Análises químicas}

As análises do teor de umidade, cinzas e proteína bruta foram realizadas conforme técnicas descritas por CUNNIFF ${ }^{4}$.

Os lipídios totais foram determinados segundo BLIGH e $\mathrm{DYER}^{2}$, na proporção de metanol, clorofórmio e água, respectivamente $(2: 2: 1,8 \mathrm{v} / \mathrm{v} / \mathrm{v})$.

A transesterificação dos ácidos graxos dos lipídios totais foi realizada segundo procedimento de JOSEPH e ACKMAN ${ }^{11}$.

\subsection{Análise cromatográfica dos ésteres metílicos de ácidos graxos}

Os ésteres de ácidos graxos foram separados em cromatógrafo gasoso Varian, equipado com detector de ionização de chama e coluna capilar de sílica fundida CP (Select FAME CP - 7420) (100 m de comprimento, 0,25 mm de diâmetro interno e filme de $0,25 \mu \mathrm{m}$ de cianopropil). O volume injetado foi de $1,0 \mu \mathrm{L}$, utilizando razão de split $1: 80$, sendo as temperaturas do injetor e detector de 220 e $240{ }^{\circ} \mathrm{C}$, respectivamente, enquanto a temperatura da coluna foi de $165{ }^{\circ} \mathrm{C}$ durante 18 minutos e elevada a $235{ }^{\circ} \mathrm{C}$ com taxa de $4{ }^{\circ} \mathrm{C} /$ min e mantida por 25 minutos.

A identificação dos ácidos graxos foi efetuada através da comparação dos tempos de retenção com padrões Sigma (EUA), por co-eluição "spiking" de padrões junto com a amostra e comparativamente com valores de ECL (Equivalent Chain Lenght). As porcentagens de ácidos graxos foram determinadas através da integração das áreas dos picos pelo Software Varian Workstation Star, versão 5.0 e os dados expressos em porcentagens de área relativa..

\subsection{Análise estatística}

Os resultados foram submetidos à análise de variância (ANOVA) a $5 \%$ de probabilidade, pelo teste de Tukey, através do programa Statistica versão 5,0 ${ }^{19}$.

\section{Resultados e discussão}

\subsection{Composição centesimal}

Na Tabela 1 estão apresentados os valores de umidade, proteína bruta, lipídios totais e cinzas para farinha de cabeça de tilápia. 
Tabela 1. Composição centesimal da farinha de cabeça de tilápia.

\begin{tabular}{cc}
\hline Composição química (\%) & Farinha de cabeça de tilápia \\
\hline Umidade & $6,0 \pm 0,09$ \\
Cinzas & $19,4 \pm 0,14$ \\
Proteína & $38,4 \pm 0,12$ \\
Lipídios totais & $35,5 \pm 0,16$ \\
\hline Os resultados são médias de 9 replicatas com as respectivas estimativas de desvio padrão.
\end{tabular}

O teor de umidade encontrado para a farinha de cabeça de tilápia foi de 6,0\%. Este valor está de acordo com o Regulamento da Inspeção Industrial e Sanitária de Produtos de Origem Animal (RIISPOA) ${ }^{15}$ que descreve que o pescado não deve conter mais que $12 \%$ de umidade.

Os valores de cinzas, proteína e lipídios encontrados na farinha foram de 19,4, 38,4 e 35,5\%, respectivamente. Os elevados valores destes constituintes deve-se principalmente ao baixo teor de umidade, ou seja, no processo de elaboração da farinha houve uma redução no teor de umidade e, desta forma, ocorreu um aumento na concentraçao de proteína, lipídios totais e cinzas. O teor de proteína e lipídios totais foi superior quando comparado com alimentos integrais como feijão, arroz, carne bovina e frango ${ }^{8}$, os quais são comumente utilizados na merenda escolar. Em relação à outros resíduos de tilápias como fígado $^{21}$ e em filés ${ }^{22}$, a farinha de cabeça apresentou maiores valores de cinzas, proteína e lipídios totais.

\subsection{Análise sensorial da sopa e do caldo}

$\mathrm{Na}$ análise sensorial da sopa, de um total de 50 provadores (crianças de 10 a 12 anos), 16 atribuíram a nota máxima $(3,08)$ para a sopa e 19 crianças atribuíram a segunda maior nota $(2,91)$. No caldo, foi realizada somente a avaliação sensorial e com provadores universitários, haja vista que o sabor de peixe é acentuado e ocorre rejeição pelas crianças. No caldo, entre 50 provadores, não foi observada a nota máxima, 18 universitários atribuíram a nota de 2,74 , enquanto 16 não aprovaram o caldo, dando a nota mínima de 1,22, conforme mostra a Tabela 2 .

A baixa aceitação do caldo deve-se ao fato da sua formulação apresentar somente farinha de cabeça, água e sal, ou seja, foi formulado sem a adição de vegetais e especiarias que mascaram o gosto de peixe.

\subsection{Composição centesimal da sopa e controle}

No controle (sopa sem farinha), foi determinada a composição química para avaliar comparativamente o efeito da adição da farinha em relação aos componentes da composição centesimal (umidade, cinzas, proteína e lipídios totais) da sopa.

A Tabela 3 apresenta a composição centesimal da sopa e do controle.
Tabela 3. Composição centesimal da sopa e controle.

\begin{tabular}{lrc}
\hline Constituintes (\%) & Sopa & Controle \\
\hline Umidade & $81,67^{\mathrm{a}} \pm 0,92^{\mathrm{a}}$ & $85,45^{\mathrm{b}} \pm 0,89^{\mathrm{b}}$ \\
Cinzas & $2,12^{\mathrm{a}} \pm 0,10^{\mathrm{a}}$ & $1,69^{\mathrm{b}} \pm 0,03^{\mathrm{b}}$ \\
Proteína & $2,96^{\mathrm{a}} \pm 0,14^{\mathrm{a}}$ & $0,87^{\mathrm{b}} \pm 0,05^{\mathrm{b}}$ \\
Lipídios totais & $4,26^{\mathrm{a}} \pm 0,19^{\mathrm{a}}$ & $2,93^{\mathrm{b}} \pm 0,20^{\mathrm{b}}$ \\
\hline Os resultados são médias de triplicatas com as respectivas estimativas de desvio padrão.
\end{tabular}

Letras diferentes na mesma linha indicam diferença mínima significativa em nível de $5 \%$ de probabilidade.

A adição de farinha aumentou com diferença significativa os teores de cinzas, proteína e lipídios totais na sopa em relação ao controle. Os valores de cinzas aumentaram aproximadamente $25 \%$, enquanto os valores de proteína bruta e lipídios totais aumentaram em 240 e $45 \%$, respectivamente.

\subsection{Composição em ácidos graxos da farinha, sopa e controle}

A composição de ácidos graxos dos lipídios totais da farinha, sopa e controle são apresentados na Tabela 4.

Foi encontrado um total de 36 componentes nos lipídios totais para a farinha, sendo 31 componentes identificados como ácidos graxos, dentre estes os majoritários foram os ácidos palmítico (16:0), oléico (18:1n-9) e LNA, estes ácidos também foram predominantes na fração lipídica de cabeças de tilápia juvenis encontrados por VISENTAINER et al. ${ }^{20} \mathrm{e} \mathrm{em}$ filés de tilápia adultas ${ }^{22}$.

$\mathrm{Na}$ sopa, foram identificados 31 ácidos graxos, sendo estes os mesmos daqueles existentes na farinha, no entanto houve alteração na porcentagem de todos os ácidos analisados devido à metodologia da normalização de área. No controle, foi identificado um total de 22 ácidos graxos, sendo comuns aos encontrados na farinha e na sopa, no entanto um total de 9 ácidos não foram detectados.

As porcentagens da maioria dos ácidos graxos encontrados na sopa e no controle, apresentaram diferença significativa, exceto para os ácidos graxos 18:0, 18:1n-9, 18:1n-5, 20:0 e 20:1n-9. Os ácidos graxos de cadeia longa EPA e DHA não foram detectados no controle, isto ocorreu devido à inexistência de ingredientes de origem animal utilizados na sua preparação.

A inclusão da farinha na elaboração da sopa estabeleceu um aumento na porcentagem de todos os ácidos ômega-3. A porcentagem relativa do ácido LNA foi superior na sopa em relação ao controle, no entanto em relação à porcentagem relativa de LA, houve uma inversão, com maior porcentagem para o controle. Isto ocorreu devido à contribuição proveniente do óleo de soja que é uma fonte rica em ácido linoléico ${ }^{12}$.

O controle apresentou um elevado teor do ácido linoléico (48,22\%), quando comparado com a sopa $(42,63 \%)$ e assim

Tabela 2. Notas Atribuídas aos produtos elaborados com farinha de cabeça de tilápia.

\begin{tabular}{|c|c|c|c|c|c|c|c|c|c|c|}
\hline \multirow[b]{2}{*}{$\mathrm{n}^{\circ}$ de provadores } & \multicolumn{6}{|c|}{ Sopa } & \multicolumn{4}{|c|}{ Caldo } \\
\hline & 16 & 19 & 03 & 07 & 03 & 02 & 18 & 8 & 8 & 16 \\
\hline Nota & 3,08 & 2,91 & 2,74 & 2,55 & 1,58 & 1,22 & 2,74 & 2,55 & 1,58 & 1,22 \\
\hline
\end{tabular}


Tabela 4. Composição de ácidos graxos nos lipídios totais da farinha, sopa e controle.

\begin{tabular}{|c|c|c|c|}
\hline Ácidos graxos & Farinha & Sopa & Controle \\
\hline $14: 0$ & $2,54 \pm 0,13$ & $0,78^{a} \pm 0,07$ & $0,09^{b} \pm 0,03$ \\
\hline $14: 1 n-9$ & $0,15 \pm 0,02$ & $0,04 \pm 0,28$ & ND \\
\hline $15: 0$ & $0,21 \pm 0,02$ & $0,07 \pm 0,08$ & ND \\
\hline $16: 0$ & $23,60 \pm 0,11$ & $10,41^{\mathrm{a}} \pm 0,10$ & $5,93^{\mathrm{b}} \pm 0,47$ \\
\hline $16: 1 n-9$ & $0,73 \pm 0,02$ & $0,21^{\mathrm{a}} \pm 0,01$ & $0,04^{\mathrm{b}} \pm 0,01$ \\
\hline $16: 1 \mathrm{n}-7$ & $5,77 \pm 0,31$ & $1,36^{\mathrm{a}} \pm 0,03$ & $0,16^{\mathrm{b}} \pm 0,02$ \\
\hline $16: 1 \mathrm{n}-5$ & $0,15 \pm 0,24$ & $0,21 \pm 0,01$ & ND \\
\hline $17: 0$ & $0,34 \pm 0,08$ & $0,13^{\mathrm{a}} \pm 0,03$ & $0,05^{\mathrm{b}} \pm 0,03$ \\
\hline $17: 1 n-9$ & $0,27 \pm 0,09$ & $0,11^{\mathrm{a}} \pm 0,06$ & $0,04^{\mathrm{b}} \pm 0,02$ \\
\hline $18: 0$ & $6,77 \pm 0,08$ & $3,59 \pm 0,50$ & $2,85 \pm 0,10$ \\
\hline $18: 1 n-9$ & $35,09 \pm 1,23$ & $33,98 \pm 0,31$ & $35,24 \pm 0,27$ \\
\hline $18: 1 n-7$ & $2,32 \pm 0,16$ & $0,03^{a} \pm 0,03$ & $0,86^{\mathrm{b}} \pm 0,04$ \\
\hline $18: 1 n-5$ & $0,13 \pm 0,01$ & $0,53 \pm 0,04$ & $0,46 \pm 0,03$ \\
\hline $18: 2 n-6$ (LA) & $11,60 \pm 0,35$ & $42,63^{a} \pm 0,25$ & $48,22^{\mathrm{b}} \pm 0,11$ \\
\hline $18: 3 n-6$ & $0,83 \pm 0,03$ & $0,23^{a} \pm 0,02$ & $0,11^{\mathrm{b}} \pm 0,01$ \\
\hline 18:3n-3 (LNA) & $0,97 \pm 0,03$ & $1,37^{\mathrm{a}} \pm 0,01$ & $1,06^{\mathrm{b}} \pm 0,06$ \\
\hline $20: 0$ & $0,22 \pm 0,02$ & $0,32 \pm 0,03$ & $0,92 \pm 0,02$ \\
\hline $20: 1 n-9$ & $1,75 \pm 0,10$ & $0,66 \pm 0,04$ & $0,32 \pm 0,05$ \\
\hline $21: 0$ & $0,35 \pm 0,03$ & $0,09^{\mathrm{a}} \pm 0,06$ & $1,88^{\mathrm{b}} \pm 0,07$ \\
\hline $20: 2 n-6$ & $0,57 \pm 0,05$ & $0,19 \pm 0,03$ & TR \\
\hline $20: 3 n-6$ & $0,66 \pm 0,07$ & $0,21 \pm 0,03$ & TR \\
\hline $20: 3 n-3$ & $0,07 b \pm 0,02$ & $0,61 \pm 0,03$ & ND \\
\hline $22: 1 n-9$ & $1,22 \pm 0,04$ & $0,36 \pm 0,08$ & $\mathrm{TR}$ \\
\hline $22: 2 n-6$ & $0,07 \pm 0,05$ & $0,05 \pm 0,02$ & ND \\
\hline $20: 4 n-6$ (AA) & $0,05 \pm 0,05$ & $0,02 \pm 0,02$ & ND \\
\hline 20:5n-3 (EPA) & $0,03 \pm 0,14$ & $0,04 \pm 0,07$ & ND \\
\hline $22: 0$ & $0,23 \pm 0,03$ & $\mathrm{TR}$ & $0,50 \pm 0,06$ \\
\hline $24: 0$ & $0,04 \pm 0,03$ & $0,23^{a} \pm 0,02$ & $0,16^{\mathrm{b}} \pm 0,01$ \\
\hline $24: 1 n-9$ & $0,68 \pm 0,07$ & $0,23^{\mathrm{a}} \pm 0,07$ & $0,03^{\mathrm{b}} \pm 0,01$ \\
\hline $22: 4 n-3$ & $0,59 \pm 0,07$ & $0,24 \pm 0,03$ & ND \\
\hline 22:6n-3 (DHA) & $0,65 \pm 0,06$ & $0,24 \pm 0,04$ & ND \\
\hline AG não identificados & $1,35 \pm 0,12$ & $0,69 \pm 0,04$ & $1,06 \pm 0,02$ \\
\hline AGPI & $16,02 \pm 0,41$ & $45,83^{\mathrm{a}} \pm 0,27$ & $49,39^{b} \pm 0,13$ \\
\hline AGMI & $48,26 \pm 1,31$ & $37,72 \pm 0,44$ & $37,15 \pm 0,28$ \\
\hline AGS & $34,30 \pm 0,21$ & $15,62^{\mathrm{a}} \pm 0,53$ & $12,38^{\mathrm{b}} \pm 0,49$ \\
\hline n-6 & $13,78 \pm 0,37$ & $43,33^{a} \pm 0,23$ & $48,33^{\mathrm{b}} \pm 0,11$ \\
\hline n-3 & $2,24 \pm 0,17$ & $2,25^{\mathrm{a}} \pm 0,09$ & $1,06^{\mathrm{b}} \pm 0,06$ \\
\hline AGPI/AGS & $0,47 \pm 0,01$ & $2,93 \pm 0,01$ & $3,99 \pm 0,16$ \\
\hline$n-6 / n-3$ & $6,15 \pm 0,50$ & $17,33^{a} \pm 0,72$ & $45,59^{b} \pm 2,58$ \\
\hline
\end{tabular}

Médias de 9 replicatas com as respectivas estimativas de desvio padrão. Letras diferentes na mesma linha indicam diferença mínima significativa em nível de $5 \%$ de probabilidade. Abreviaturas: $\mathrm{ND}=$ não detectado; TR (traço) = área mínima menor que 0,02; AG = ácido graxo; AGPI = somatório dos ácidos graxos poliinsaturados; AGMI = somatório dos ácidos graxos monoinsaturados; AGS = somatório dos ácidos graxos saturados; n- 6 e n-3 = somatórios dos ácidos graxos da série n-6 e n-3, respectivamente; AGPI/AGS = razão entre o somatório dos ácidos poliinsaturados e saturados; e n-6/n-3 = razão entre o somatório dos ácidos da série n-6 e n-3.

elevou o valor do somatório dos ácidos n-6 (48,33\%). No entanto, em relação ao somatório de ácidos graxos n-3, a sopa apresentou valor de $2,25 \%$, valor muito superior ao encontrado no controle $1,06 \%$.

Conforme o Departamento de Saúde da Inglaterra ${ }^{9}$, os alimentos com valores da razão AGPI/AGS superiores a 0,45 são benéficos na prevenção de doenças cardiovasculares. Neste sentido, a sopa com valor para a razão AGPI/AGS de 2,93 é considerada um alimento benéfico para a saúde humana.

\section{Conclusões}

A farinha de cabeça de tilápia é um resíduo que apresenta um excelente valor nutritivo, devido ao seu elevado teor protéi- co, cinzas (minerais) e altamente energética, devido ao elevado teor de lipídios.

A inclusão da farinha na elaboração da sopa aumentou a concentração de todos os ácidos graxos ômega-3, especialmente dos ácidos LNA, EPA e DHA.

A farinha elaborada sob a forma de sopa apresentou excelente aceitação pelos alunos do ensino fundamental e, desta forma, pode ser recomendada para a merenda escolar.

Sendo assim, cabeças de tilápia, podem ser utilizadas como matéria-prima de baixo custo para produção de produtos alimentícios, agregando assim maior valor aos resíduos de peixes e diminuindo a poluição ambiental. 


\section{Referências bibliográficas}

1. BELDA, M. C. R.; POURCHET-CAMPOS, M. A. Ácidos graxos essenciais em nutrição: uma visão atualizada. Ciência e Tecnologia de Alimentos, v. 11, n. 1, p. 5-35, 1991.

2. BLIGH, E. G.; DYER, W. J. A rapid method of total lipid extraction and purification. Canadian Journal of Biochemistry, v. 37, n. 18, p. 911-917, 1959 .

3. CHAVES, J. B. P. Avaliação sensorial de alimentos - Métodos de Análises. Viçosa. Editora da Universidade Federal de Viçosa, 69 p., 1980.

4. CUNNIFF, P. A. Official methods of Analysis of AOAC International. 16th ed. Arlington: Association of Official Analytical Chemists. 1998. CD-Rom.

5. DUSTAN, J. A., ROPER, J., MitOUlAS, L. The effects of supplementation with fish oil during pregnancy on breast milk immunoglobulin A, soluble CD14, cytokine levels and fatty acid composition. Clinical \& Experimental Allergy, v. 34, n. 8, p. 1237-1242, 2004.

6. DYEBERG, J.; BANG, H. O. Homeostatic function and platelet polyunsaturated fatty acids in Eskimos. Lancet, v. 2, p. 433-435, 1979.

7. FAO - Food and Agriculture Organization of the United Nations. Estatísticas da Pesca. Roma. v. 91, p. 141, 2001.

8. FRANCO, G. V. E. Tabela de composição química de alimentos. 9. edição. São Paulo: Livraria Atheneu, p. 324, 2001.

9. HMSO, (1994). Report on Health and Social Subjects. Department of Health. Nutritional aspects of cardiovascular disease. England, London, v. 46, p. 37-46, 1984.

10. IBAMA - Instituto brasileiro do meio ambiente e dos recursos naturais renováveis. Produção brasileira da aqüicultura de água doce, por estado e espécie, para o ano de 2003. 2004. Disponível em http://www.presidencia.gov.br/seap/. Acesso em 15 Junho, 2005.

11. JOSEPH, J. D.; ACKMAN, R. G. Capillary column gas chromatography method for analysis of encapsulated fish oil and fish oil ethyl esters: collaborative study. Journal of AOAC International, v. 75, n. 5, p. 488-506, 1992.
12. MARTIN, C. A. et al. Trans Polyunsaturated fatty acids contents in Brazilian refined soybean oil. Analytical Sciences, v. 22, n. 4, p. 631-633, 2006.

13. OETTERER, M. Industrialização do pescado cultivado. Guaíba, RS: Livraria e editora Agropecuária, 2002.

14. PESSATTI, M. L. 2001. Aproveitamento dos subprodutos do pescado. Meta 11. Relatório Final de Ações Prioritárias ao Desenvolvimento da Pesca e Aqüicultura no Sul do Brasil, Convênio Ministério da Agricultura, Pecuária e Abastecimento (MAPA), Universidade do Vale do Itajaí, MA/SARC, n. 003/2000

15. RIISPOA, 1997 - Regulamento da Inspeção Industrial e Sanitária de Produtos de Origem animal. Ministério da Agricultura, Pecuária e Abastecimento. Seção II - Derivado do Pescado, Artigo 466.

16. SIMOPOULOS, A. P.; LEAF, A.; SALEM, N. Essentially and recommended dietary intakes for omega- 6 and omega-3 fatty acids. Annals of Nutrition and Metabolism, v. 43, p. 127-130, 1999.

17. SIMOPOULOS, A. P. Omega-3 fatty acids in inflammation and autoimmune diseases. Journal of the American College of Nutrition, v. 21, n. 6, p. 495-505, 2002.

18. SOUZA, N. E. et al. Composição química, perfil de ácidos graxos e quantificação dos ácidos $\alpha$-linolênico, eicosapentaenóico e docosahexaenóico em vísceras de tilapias (Oreochromis niloticus). Acta. Scientiarum Tecnology, v. 27, n. 1, p. 7376, 2005.

19. STATISTICA. Statistica $\mathbf{5 . 0}$ software. Stasoft, Tucksa, 1995.

20. VISENTAINER, J. V. et al. Quantificação de ácidos graxos ômega-3 (LNA, EPA E DHA), caracterização físico -químico e composição em ácidos graxos em cabeças de tilápias jovens. In: Congresso Brasileiro de Ciência e Tecnologia de Alimentos, 2000, Fortaleza: SBCTA/UFC, p. 5-221.

21. VISENTAINER, J. V. et al. Composição físico-química e de ácidos graxos em fígados de tilápias (Oreochromis niloticus) submetidas as fornecimento de rações diferenciadas à base de óleos de girassol e linhaça. Anais da Associação Brasileira de Química, São Paulo, v. 51, n.1, p. 18-21, 2003.

22. VISEnTAINER, J. V. et al. Composição química e de ácidos graxos em tilápias (Oreochromis niloticus) submetidas à dieta prolongada. Revista Nacional da Carne, n. 319, p. 152-154, 2003. 